\title{
Surface Impedance and Bulk Band Geometric Phases in One-Dimensional Systems
}

\author{
Meng Xiao, Z. Q. Zhang, and C. T. Chan ${ }^{*}$ \\ Department of Physics and Institute for Advanced Study, \\ The Hong Kong University of Science and Technology, \\ Clear Water Bay, Kowloon, Hong Kong, China
}

(Received 7 January 2014; revised manuscript received 17 February 2014; published 25 April 2014)

\begin{abstract}
Surface impedance is an important concept in classical wave systems such as photonic crystals (PCs). For example, the condition of an interface state formation in the interfacial region of two different one-dimensional PCs is simply $Z_{\mathrm{SL}}+Z_{\mathrm{SR}}=0$, where $Z_{\mathrm{SL}}\left(Z_{\mathrm{SR}}\right)$ is the surface impedance of the semiinfinite PC on the left-hand (right-hand) side of the interface. Here, we also show a rigorous relation between the surface impedance of a one-dimensional PC and its bulk properties through the geometrical (Zak) phases of the bulk bands, which can be used to determine the existence or nonexistence of interface states at the interface of the two PCs in a particular band gap. Our results hold for any PCs with inversion symmetry, independent of the frequency of the gap and the symmetry point where the gap lies in the Brillouin zone. Our results provide new insights into the relationship between surface scattering properties, the bulk band properties, and the formation of interface states, which in turn can enable the design of systems with interface states in a rational manner.
\end{abstract}

DOI: 10.1103/PhysRevX.4.021017

\section{INTRODUCTION}

Impedance is a very important and useful concept in wave physics because it is the parameter that governs how a wave is scattered or reflected when it encounters an interface. As such, it characterizes how a material couples with waves coming from outside. On the other hand, the bulk band structure characterizes how waves can travel inside a periodic system. These quantities should be related in some way. We establish that, for a periodic multilayer film, commonly referred to as $1 \mathrm{D}$ photonic crystals, the surface impedance is related to the Zak phase [1] of the bulk bands. As the existence of interface states is determined by the surface impedance, this means that the existence of localized states at an interface is determined by the geometric phases of the bulk crystals. It is well known that interface states can exist in a quantum system when the topological properties of two semi-infinite systems on each side of the interface are different [2-5]. A famous example is the Su-Schrieffer-Heeger (SSH) model for polyacetylene [6-8]. In such systems, it was shown that an interface state exists when the Zak phase of the occupied band on one side of the chain is different from that on the other side, which can be obtained through gap inversion [9-11]. The purpose of this work is to find a general

\footnotetext{
*Corresponding author. phchan@ust.hk

Published by the American Physical Society under the terms of the Creative Commons Attribution 3.0 License. Further distribution of this work must maintain attribution to the author(s) and the published article's title, journal citation, and DOI.
}

Subject Areas: Metamaterials, Optics, Photonics

connection between the existence of an interface state in a photonic system and the bulk band topological properties as well as the surface impedances of the two systems on each side of the boundary. The analog between photonic systems and quantum systems was discussed recently [12-15]. Based on this analog, the Zak phase can also be defined in photonic crystals (PCs). For 1D binary PCs, we find a rigorous relation that relates the existence of an interface state to the sum of all Zak phases below the gap on either side of the interface. This relation holds for any 1D PCs with inversion symmetry, including those with graded refractive indices. Similar to the "bulk-edge correspondence" found in topological insulators [2-4], the "bulkinterface correspondence" found here provides not only a tool to determine the existence of interface states in a photonic system but also the possibility of designing a photonic system with interface states appearing in a set of prescribed gaps.

\section{RESULTS}

\section{A. Impedances and Zak phases of 1D photonic crystals and their relationship}

Let us consider a dielectric $\mathrm{AB}$ layered structure as shown in Fig. 1(a). A plane wave from free-space incidents normally on the semi-infinite $1 \mathrm{D}$ PC on the right and the reflection coefficient of the electric field $E_{x}$ is given by $r_{R}$. When the frequency of the incident wave is inside the band gap of this system, the incident wave will be totally reflected, and we have $r_{R}=e^{i \phi_{R}}$, where $\phi_{R}$ is the reflection phase. We define a surface impedance $Z_{\mathrm{SR}}$ of the semiinfinite PC as the ratio of the total electric field to the total 
magnetic field on the right-hand side of the boundary, i.e., $Z_{\mathrm{SR}}=E_{x}\left(z=0^{+}\right) / H_{y}\left(z=0^{+}\right)$, where $\mathrm{z}=0$ defines the boundary. The impedance $Z_{\mathrm{SR}}$ and the reflection coefficient $r_{R}$ are related by

$$
Z_{\mathrm{SR}}=\frac{1+r_{R}}{1-r_{R}} Z_{0}
$$

where $Z_{0}$ is the vacuum impedance. Inside a band gap, $Z_{\mathrm{SR}}$ is a pure imaginary number and we can write $Z_{\mathrm{SR}} / Z_{0} \equiv i \varsigma_{R}$, where $\varsigma_{R}$ is a real number. The reflection phase can hence be expressed as $\phi_{R}=\pi-2 \arctan \left(\varsigma_{R}\right)$. Let us now put another PC on the left of the interface, and we denote the impedance and the reflection phase of the left PC by $Z_{\mathrm{SL}}$ and the reflection coefficient $r_{L}$. The condition for the presence of an interface state is simply $Z_{\mathrm{SR}}+Z_{\mathrm{SL}}=0$. According to Eq. (1), this condition implies that $\left(1+r_{R}\right) /\left(1-r_{R}\right)+\left(1+r_{L}\right) /\left(1-r_{L}\right)=0$, which is equivalent to $r_{R} r_{L}=1$ or $\phi_{R}+\phi_{L}=2 m \pi(m \in \mathbb{N})$. A simple way to obtain an interface state is to construct a system in which the surface impedances on the two sides are opposite in sign. A similar condition has been reported for $2 \mathrm{D}$ PCs $[16,17]$. But the question is then, how can we design or control the value of the surface impedance? We show that the sign of the surface impedance for frequencies inside a band gap is, in fact, determined by the geometrical phase of the bulk bands. In the following, we derive a rigorous relation between the surface impedance and the Zak phase of the PC.

The band structure of a dielectric binary PC shown in Fig. 1(a) can be obtained from the following relation [18]:

(a)
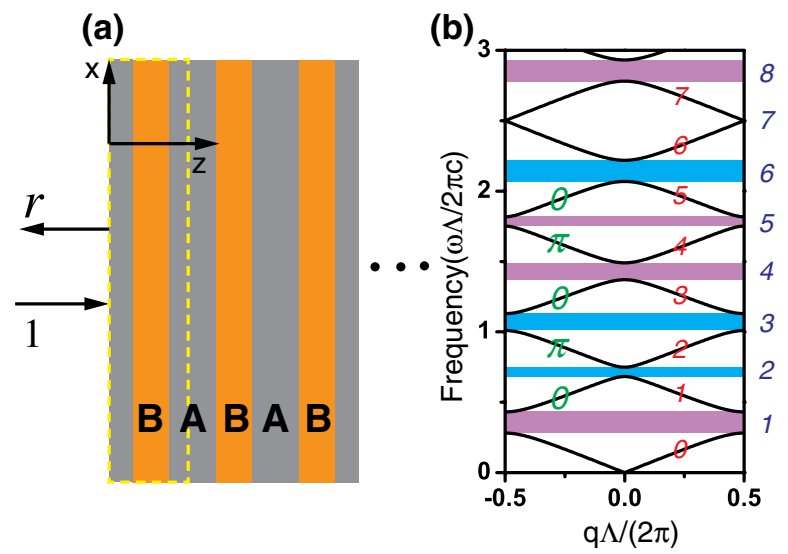

FIG. 1. (a) Plane wave incidents normally on an AB layered structure. The reflection coefficient of the electric field is given by $r$. The yellow dashed line marks the unit cell we consider. (b) The band structure of the PC (solid black curve) with parameters given by $\varepsilon_{a}=4, \mu_{a}=\varepsilon_{b}=\mu_{b}=1, d_{a}=0.4 \Lambda$, and $d_{b}=0.6 \Lambda$, where $\Lambda$ is the length of the unit cell. The magenta strip represents the gap with $\varsigma>0$, while the cyan strip represents the gap with $\varsigma<0$. The Zak phase of each individual band is labeled in green, and the numbers of the bands and gaps are listed with red and blue labels, respectively.

$$
\begin{aligned}
\cos (q \Lambda)= & \cos k_{a} d_{a} \cos k_{b} d_{b} \\
& -\frac{1}{2}\left(\frac{z_{a}}{z_{b}}+\frac{z_{b}}{z_{a}}\right) \sin k_{a} d_{a} \sin k_{b} d_{b},
\end{aligned}
$$

where $k_{i}=\omega n_{i} / c, n_{i}=\sqrt{\mu_{i} \varepsilon_{i}}, z_{i}=\sqrt{\mu_{i} / \varepsilon_{i}},(i=a$ or $b)$; $d_{a}, d_{b}$, and $\Lambda=d_{a}+d_{b}$ are the widths of slabs $A$ and $B$ and the unit cell, respectively; and $q$ is the Bloch wave vector. Here, $c$ denotes the wave speed in vacuum, $\varepsilon_{a}, \varepsilon_{b}$, $\mu_{a}$, and $\mu_{b}$ are the relative permittivity and permeability of slabs $A$ and $B$, respectively. The band structure for the parameters $\varepsilon_{a}=4, \mu_{a}=\varepsilon_{b}=\mu_{b}=1, d_{a}=0.4 \Lambda$, and $d_{b}=0.6 \Lambda$ is shown in Fig. 1(b). For convenience of discussion, we have numbered the pass bands and band gaps, independent of whether a gap is closed or not. It is easy to show that the midgap positions (or the crossing points when two bands meet) of the $\mathrm{PC}$ are at $\omega_{m}=$ $m \pi c /\left(n_{a} d_{a}+n_{b} d_{b}\right)$ (see Appendix A). For each isolated (no crossing) band $n$, we define the Zak phase as [12-14,19]

$$
\theta_{n}^{\mathrm{Zak}}=\int_{-\pi / \Lambda}^{\pi / \Lambda}\left[i \int_{\text {unit cell }} d z \varepsilon(z) u_{n, q}^{*}(z) \partial_{q} u_{n, q}(z)\right] d q,
$$

where $i \int_{\text {unit cell }} d z \varepsilon(z) u_{n, q}^{*}(z) \partial_{q} u_{n, q}(z)$ is the Berry connection, $\varepsilon(z)$ denotes the dielectric function, and $u_{n, q}(z)$ is the periodic-in-cell part of the Bloch electric field eigenfunction of a state on the $n$th band with wave vector $q$, i.e., $E_{x ; n, q}(z)=u_{n, q}(z) \exp (i q z)$. For the case of a binary PC, the function $u_{n, q}(z)$ can be obtained analytically from the transfer-matrix method [18] (see Appendix B). The 1D system with inversion symmetry always has two inversion centers and the Zak phase is quantized at either 0 or $\pi$ if the origin is chosen to be one of the inversion center [1]. If the Zak phase equals $0(\pi)$ relative to one inversion center, it must be $\pi(0)$ relative to the other inversion center. Without loss of generality, we choose the center of slab $A$ as the origin for calculating Zak phases. If the surface of the semiinfinite $\mathrm{PC}$ is also chosen at the same origin, i.e., the center of slab $A$, we find a rigorous relation between the surface impedance of the PC in the $n$th gap, i.e., $Z_{S}^{(n)} / Z_{0}=i \varsigma^{(n)}$, and the sum of Zak phases of all the isolated bands below the $n$th gap [Appendix D, Eq. (D12)]. This relationship relates the surface scattering properties and the topological properties of bulk dispersion. It can predict the existence of an interface state in a band gap and determine the location of the interface state if it exists. In addition, if we are only interested in knowing whether such a state exists in a gap, we only need to know the sign of $\varsigma^{(n)}$ on each side. The sign of $\varsigma^{(n)}$ has the following simple expression:

$$
\operatorname{sgn}\left[\varsigma^{(n)}\right]=(-1)^{n}(-1)^{l} \exp \left(i \sum_{m=0}^{n-1} \theta_{m}^{\mathrm{Zak}}\right),
$$

where the integer $l$ is the number of crossing points under the $n$th gap [in Fig. 1(b), the crossing point is at the 7th 
band gap]. The Zak phase of the lowest 0th band is determined by the sign of $\left[1-\varepsilon_{a} \mu_{b} /\left(\varepsilon_{b} \mu_{a}\right)\right]$, i.e.,

$$
\exp \left(i \theta_{0}^{\mathrm{Zak}}\right)=\operatorname{sgn}\left[1-\varepsilon_{a} \mu_{b} /\left(\varepsilon_{b} \mu_{a}\right)\right]
$$

(see Appendixes $\mathrm{C}$ and D).

We calculate the Zak phase of each isolated band (band 1-5) in Fig. 1(b) using Eq. (3). These Zak phases are shown with green letters in Fig. 1(b). According to Eq. (4), we obtain $\operatorname{sgn}[\varsigma]$ in each gap. They are marked by magenta when $\varsigma>0$ and cyan when $\varsigma<0$.

\section{B. Changing the sign of impedance by passing a topological transition point}

To have a guaranteed existence of an interface state, one needs to make sure that surface impedance on the left and right half-space is of opposite sign at one common gap frequency. One possible way (but not the only way) is to "tune the system parameters across a topological transition point," as elaborated below. To demonstrate this idea, we simply tune the parameter $\varepsilon_{a}$ used in Fig. 1(b) from 3.8 to 4.2 and keep $\mu_{a}=\mu_{b}=\varepsilon_{b}=1$ unchanged. In the meantime, we also vary $d_{a}$ and $d_{b}$ in such a way as to keep $n_{a} d_{a}+n_{b} d_{b}$ unchanged so that all of the midgap positions do not alter. In Figs. 2(b) and 2(c), we show the band structures of two PCs from the 4th gap to the 8th gap: Fig. 2(b) shows "PC1" with $\varepsilon_{a}=3.8, \varepsilon_{b}=\mu_{a}=\mu_{b}=1$, $d_{a}=0.42 \Lambda$, and $d_{b}=0.58 \Lambda$, and Fig. 2(c) shows "PC2"

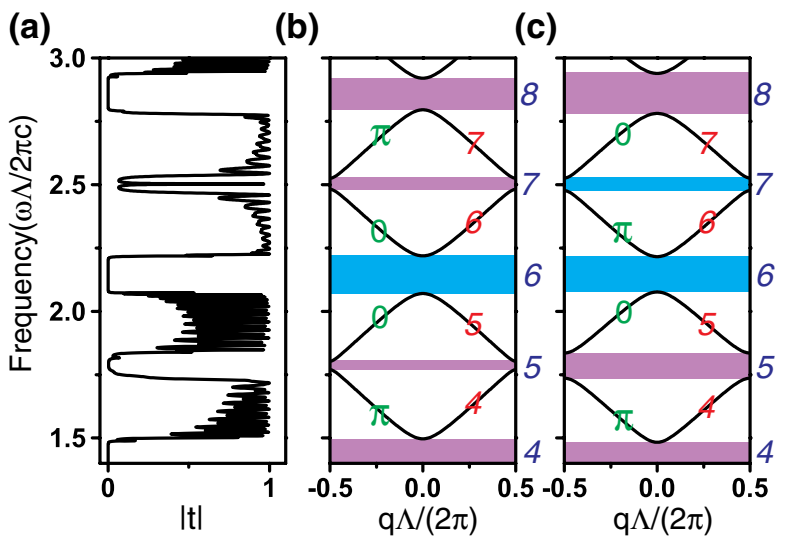

FIG. 2. (a) The transmission spectrum of a system composed of 10 unit cells of PC1 on the left-hand side and 10 unit cells of PC2 on the right-hand side in vacuum. The parameters of PC1 are given by $\varepsilon_{a}=3.8, \varepsilon_{b}=\mu_{a}=\mu_{b}=1, d_{a}=0.42 \Lambda$, and $d_{b}=0.58 \Lambda$, and the parameters of PC2 are given by $\varepsilon_{a}=4.2$, $\varepsilon_{b}=\mu_{a}=\varepsilon_{b}=1, d_{a}=0.38 \Lambda$, and $d_{b}=0.62 \Lambda$, where $\Lambda$ is the unit length of the PCs. (b),(c) The band structure (solid black curve) of PC1 and PC2. In both (b) and (c), the magenta strip represents the gap with $\varsigma>0$, while the cyan strip represents the gap with $\varsigma<0$, and the Zak phase of each individual band is also labeled in (b) and (c) in green. We note that if the gaps of the PCs on either side of the interface carry the same sign of $\varsigma$, there is no interface state. If the sign of $\varsigma$ is opposite, there must be an interface state (e.g., at a reduced frequency unit of 2.5). with $\quad \varepsilon_{a}=4.2, \quad \varepsilon_{b}=\mu_{a}=\mu_{b}=1, \quad d_{a}=0.38 \Lambda, \quad$ and $d_{b}=0.62 \Lambda$. It is interesting to see that Zak phases of all the bands below the 6th gap remain unchanged during the variation of $\varepsilon_{a}$, but the Zak phases of bands 6 and 7 in these two PCs switch with a corresponding sign change in the surface impedance in the 7th gap. When the value of $\varepsilon_{a}$ is increased from 3.8, the size of the 7th gap reduces and the crossing of band 6 and band 7 occurs when $\varepsilon_{a}=4$, at which gap 7 is closed, as shown in Fig. 1(b). When the value of $\varepsilon_{a}$ is further increased, the gap opens again and is accompanied by a change of sign in the surface impedance as well as a switch of the Zak phase in bands 6 and 7. This represents a topological phase transition, which occurs when two bands cross each other. Thus, by constructing an interface with PC1 on one side and PC2 on the other side, we should see an interface state inside gap 7. This is verified in our numerical study of the transmission spectrum of a system consisting of a slab of PC1 (with 10 unit cells) on one side and a slab of PC2 (with 10 unit cells) on the other side embedded in vacuum. Figure 2(a) clearly shows a resonance transmission due to an interface state around $\omega=5 \pi c / \Lambda$ in gap 7. Such a topological phase transition represents a classical analog of the SSH model in electronic systems [6-8] although impedance is not usually considered in electrons.

The above example is a manifestation of a topological phase transition arising from band crossing in photonic systems. It should be pointed out that the occurrence of the band crossing shown in Fig. 1(b) is by no means accidental. It can be shown rigorously (see Appendix A) that if the ratio of the optical paths in two slabs of a PC is a rational number, namely, $\alpha=n_{a} d_{a} /\left(n_{b} d_{b}\right)=m_{1} / m_{2} \in \mathbb{Q}$, where $m_{1}, m_{2} \in \mathbb{N}^{+}$, then band $m_{1}+m_{2}$ and band $m_{1}+m_{2}-1$ will cross at the frequency $\omega_{m_{1}+m_{2}}=\left(m_{1}+m_{2}\right) \pi \mathrm{c} /$ $\left(n_{a} d_{a}+n_{b} d_{b}\right)$. At this frequency, $\sin k_{a} d_{a}=\sin k_{b} d_{b}=0$, $\cos \left(k_{b} d_{b}\right)=(-1)^{l m_{2}}$, and $\cos \left(k_{a} d_{a}\right)=(-1)^{l m_{1}}$, where $l \in \mathbb{N}^{+}$, so $\cos (q \Lambda)=(-1)^{l\left(m_{1}+m_{2}\right)}$ and the gap $m_{1}+m_{2}$ will close at the center or boundary of the Brillouin zone (BZ) depending on whether $l\left(m_{1}+m_{2}\right)$ is even or odd. It is easy to see that, if the $\left(m_{1}+m_{2}\right)$ th gap is closed, so are all other gaps that are integer multiples of $m_{1}+m_{2}$. In fact, the above condition is also a necessary condition for two bands to cross (see Appendix A).

The origin of the topological phase transition shown in Fig. 2 is directly related to a special set of frequencies $\tilde{\omega}$ given by $\sin \left(n_{b} d_{b} \tilde{\omega} / c\right)=0$. It can be shown rigorously (see Appendix B) that if one of the $\tilde{\omega}$ appears inside a band, the Zak phase of the band must be $\pi$. Otherwise, it is zero. This rule applies to all bands except the 0th band, for which the Zak phase is determined by the sign of function $\left[1-\varepsilon_{a} \mu_{b} /\left(\varepsilon_{b} \mu_{a}\right)\right]$ (see Appendixes $\mathrm{C}$ and D). For the case of Fig. 2(b), $\tilde{\omega}$ appears in band 7, whereas for Fig. 2(c), $\tilde{\omega}$ appears in band 6 . Thus, the value of $\tilde{\omega}$ decreases as $\varepsilon_{a}$ is increased. For the entire band 6 of Fig. 2(b) and band 7 of Fig. 2(c), the function $\sin \left(n_{b} d_{b} \omega / c\right)$ does not change sign. 
The variation of $\tilde{\omega}$ with respect to $\varepsilon_{a}$ can be seen as follows. In Fig. 1(b) $\left(\varepsilon_{a}=4\right)$, the frequency at which two bands meet in gap 7 is $\omega_{7}=5 \pi c / \Lambda$ [see Fig. 1(b)], which is also the frequency where $\sin \left(n_{b} d_{b} \omega_{7} / c\right)=0$, i.e., $\tilde{\omega}=\omega_{7}$. When $\varepsilon_{a}$ is decreased from 4, say [Fig. 2(b)], we need to increase the value of $d_{a}$ in order to keep $n_{a} d_{a}+n_{b} d_{b}$ unchanged. Thus, the value of $n_{b} d_{b}$ is reduced accordingly, which in turn implies $\tilde{\omega}>\omega_{7}$. On the other hand, if $\varepsilon_{a}$ is increased from 4, say [Fig. 2(c)], we have $\tilde{\omega}<\omega_{7}$. Here, we have used the fact that $\tilde{\omega}$ will always appear in a pass band. This can be seen from Eq. (2) because the absolute value of the rhs of the equation at $\tilde{\omega}$ is always less than or equal to unity.

\section{Relationship between the Zak phase and the symmetry properties of the edge states}

We give a physical interpretation of the Zak phase in an isolated band by using the symmetries of the two edge states at the two symmetry points of the Brillouin zone. As we have seen, the topological property of the band structure changes every time when a band crossing occurs as $\alpha \equiv$ $n_{a} d_{a} /\left(n_{b} d_{b}\right)$ passing through a rational number, and the change can be seen from the changes in the symmetries of the edge states. As an example, let us focus on the 6th and 7th bands in Figs. 2(b) and 2(c), in which the Zak phases change by $\pi$ when $\varepsilon_{a}$ is increased from 3.8 to 4.2. These two bands are highlighted in Figs. 3(a) and 3(b) with the band edges marked by red letters. The corresonding Zak phases are shown in green. The difference in the Zak phase of each band can be understood by examing the symmetry of the absolute value of electric distribution $\left|E_{n, q}(z)\right|$ of the two edge states in the band. The black curves in Figs. 3(c), $3(\mathrm{e})$, and $3(\mathrm{~g})$ show the functions $\left|E_{n, q}(z)\right|$ in a unit cell in arbitrary units for the three edge states of PC1 at points $L$, $M$, and $N$. The black curves in Figs. 3(d), 3(f), and 3(h) correspond to the points $P, Q$, and $R$ of PC2. Here, we use the important result from Kohn [20] and Zak [1] for 1D systems with inversion symmetry, which, when generalized to a photonic system, states that the Zak phase of the $n$th band is zero if either $\left|E_{n, q=0}(z=0)\right|=\left|E_{n, q=\pi / a}(z=0)\right|=0$ or $\left|E_{n, q=0}(z=0)\right| \neq 0 ;\left|E_{n, q=\pi / a}(z=0)\right| \neq 0$. Otherwise, it is $\pi$. The blue dashed lines in Figs. 3(c)-3(h) indicate the position of the origin $(z=0)$, which is the center of slab $A$. According to this rule, it is easy to see from Figs. 3(e) and $3(\mathrm{~g})$ that the Zak phase of the 6th band of PC1 is zero as the wave functions of the points $M$ and $N$ are both nonzero at the origin, whereas the value changes to $\pi$ in PC2 because the wave function at point $Q$ becomes zero after band crossing. For the same reason, the Zak phase of the 7 th band in PC2 is also changed after band crossing. The band inversion can also be seen from the switching of two edge states across the gap. For example, the wave functions at points $L$ and $Q$ have nealy the same distribution, i.e., the wave functions are both zero at the origin and with larger amplitudes in slab $B$, whereas for points $M$ and $P$ the

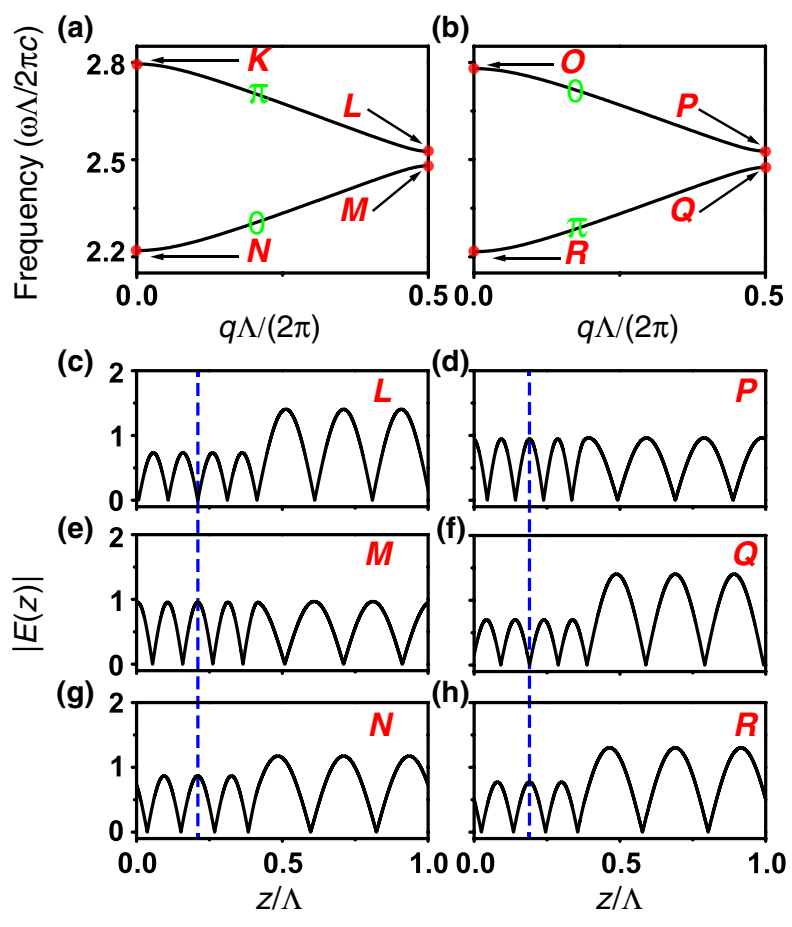

FIG. 3. (a) The band dispersion of the PC with parameters $\varepsilon_{a}=3.8, \quad \varepsilon_{b}=\mu_{a}=\mu_{b}=1, \quad d_{a}=0.42 \Lambda$, and $d_{b}=0.58 \Lambda$. (b) The band dispersion of the PC with parameters $\varepsilon_{a}=4.2$, $\varepsilon_{b}=\mu_{a}=\mu_{b}=1, d_{a}=0.38 \Lambda$, and $d_{b}=0.62 \Lambda$, where $\Lambda$ is the unit length of PCs. The Zak phase of each band in (a) and (b) is shown in green. (c)-(h) The absolute value of the electric field $E(z)$ (black solid line) of the band-edge state as a function of position z. Six band-edge states, $L, P, M, Q, N, R$, indicated with solid red circles in (a) and (b), are shown in (c)-(h), respectively. The region of slab $A$ is $\left(0, d_{a}\right)$, the left is slab $B$, and the blue dashed lines mark the center of slab $A$.

absolute values of the wave functions are both at maximum at the origin and their amplitudes are nearly the same in slab $A$ and slab $B$. However, the wave functions at points $N$ and $R$ are nearly the same, not affected by the band crossing. This is also true for points $K$ and $O$. Thus, it is precisely the switching of two edges states at gap 7 that gives rise to different Zak phases in PC1 and PC2 for both bands 6 and 7. Similar behavior has been reported in the electronic system [9-11].

\section{Relationship between the sign of impedance and the symmetry properties of the edge states}

The sign of the imaginary part of the surface impedance, i.e., $\varsigma$, can also be related to the symmetries of the two edge states. It is well known that the amplitude of the wave function of the band-edge states at the origin $(z=0)$ is either zero or maximum [21] as is also shown in Fig. 3 (see a proof in Appendix C). For convenience, we name the wave function with zero amplitude at the origin as $A$ (antisymmetric) state and the other as $S$ (symmetric) state. For the $A$ state, the electric field is zero at the boundary of 
the $\mathrm{PC}$, which is equivalent to a perfect electric conductorlike boundary condition, while the electric field is maximum at the boundary for the $S$ state, which gives a perfect magnetic conductor-like boundary condition. Since two edge states across a gap are orthogonal, they always belong to different symmetries. With this definition, as can be easily seen from Figs. 3(c)-3(h), points $L$ and $Q$ belong to type $A$, whereas points $P, M, N$, and $R$ belong to type $S$. If a reflection measurement is done at the frequency of the type$A$ state, we must have $r=-1$, corresponding to a reflection phase $\phi=\pi$. On the other hand, if the measurement is done at the frequency of the type- $S$ state, we have $r=1$ and $\phi=0$ or $2 \pi$. From the relation $\phi=\pi-2 \arctan (\varsigma)$, it can be shown that, for a gap with state $\mathrm{A}$ at the lower edge, the function $\varsigma$ has a value 0 at the lower edge and decreases monotonically to $-\infty$ as the upper edge is approached. For a gap with state $S$ at the lower edge, the function $\varsigma$ decreases monotonically from $\infty$ to 0 as the upper edge is approached (see Appendix D). Thus, the sign of $\varsigma$ in a gap is determined solely by the type of state at the lower edge (or upper edge, since these two states are orthogonal) of the gap, and if two states at the lower edges of the common gap belong to different types, an interface state must exist inside the gap.

\section{E. Existence of interface states}

As we mentioned earlier, the occurrence of band crossing at a particular gap (say, the $n$th gap) appears simultaneously for all gaps that are integer multiples of the $n$th gap. However, we emphasize that "gap inversion" is just one way, but not the only way, to achieve an interface state. As an example, we consider a system consisting of 10 unit cells of "PC3" $\left(\varepsilon_{b}=3.5, \varepsilon_{a}=\mu_{a}=\mu_{b}=1, d_{a}=0.35 \Lambda\right.$, and $d_{b}=0.65 \Lambda$ ) on the left and 10 unit cells of "PC4" $\left(\mu_{b}=6, \varepsilon_{a}=\mu_{a}=\varepsilon_{b}=1, d_{a}=0.6 \Lambda\right.$, and $\left.d_{b}=0.4 \Lambda\right)$ on the right embedded in vacuum. The corresponding band structures are shown in Figs. 4(b) and 4(c) for PC3 and $\mathrm{PC} 4$, respectively. There are six overlapping gaps in the frequency range in which we are interested. The transmission spectrum of the system is shown in Fig. 4(a). We find three interface states in gaps 1,2, and 5. The existence of interface states in these gaps is not due to band inversion. However, their existence can still be predicted by Eq. (4). To demonstrate this, we calculate the Zak phase of each isolated band in PC3 and PC4 using Eq. (3). The results are shown in Figs. 4(b) and 4(c) with green letters. The sign of the imaginary part of the surface impedance, i.e.,sgn[ $\varsigma]$, of each gap can now be obtained from Eq. (4). As before, we mark the $\varsigma>0$ gaps with magenta and the $\varsigma<0$ gaps with cyan. According to the condition of an interface state, i.e., $\varsigma_{L}+\varsigma_{R}=0$, the interface states exist only in gaps 1,2 , and 5 in which $\varsigma_{L}$ and $\varsigma_{R}$ have different signs. This is consistent with the result of the transmission study shown in Fig. 4(a). (a)

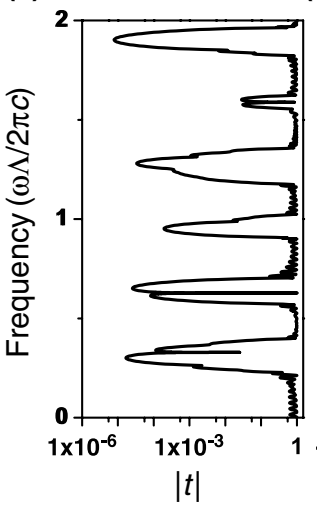

(b)

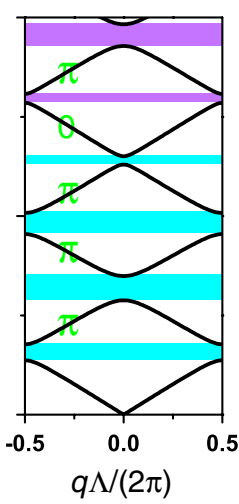

(c)

FIG. 4. (a) The transmission spectrum of the system composed of 10 unit cells of PC3 on the left-hand side and 10 unit cells of PC4 on the right-hand side in vacuum. The parameters of PC3 are given by $\varepsilon_{b}=3.5, \quad \varepsilon_{a}=\mu_{a}=\mu_{b}=1, \quad d_{a}=0.35 \Lambda$, and $d_{b}=0.65 \Lambda$, and the parameters of PC4 are given by $\mu_{b}=6$, $\varepsilon_{a}=\mu_{a}=\varepsilon_{b}=1, d_{a}=0.6 \Lambda$, and $d_{b}=0.4 \Lambda$, where $\Lambda$ is the unit length of the PCs. (b),(c) The band structure (solid black curve) of PC3 and PC4. In both (b) and (c), the magenta strip represents the gap with $\varsigma>0$, while the cyan strip represents the gap with $\varsigma<0$, and the Zak phase of each individual band is also labeled in (b) and (c) in green. Whenever two gaps with different character (different sign of $\varsigma$ ) have a common region, there will be an interface state.

\section{F. Generalization to other waves}

Finally, we want to stress that the results obtained above for PCs also hold for other one-dimensional systems with inversion symmetry, such as acoustic waves. Because of inversion symmetry, the wave functions at two edges of an isolated band can be either symmetric with a maximum amplitude or antisymmetric with zero amplitude. Thus, the symmetry properties of these two edge states determine the Zak phase of the band. From Eq. (4), the sign of the imaginary part of the surface impedance $\varsigma$ can be determined. An interface state can then be created by constructing an interface from two semi-infinite systems with opposite signs in $\varsigma$. The validity of Eq. (4) is also not limited to the binary layer structure considered in this work. In fact, Eq. (4) also holds when the relative permittivity and permeability are continuously varying functions of position as long as the inversion symmetry is kept, and the lattice constants of the left and right periodic systems do not need to be equal. Examples are given in Appendix E.

We mention that the electric field is taken as the scalar field in this work. If the magnetic field is chosen as the scalar field, Eq. (4) still holds. The sign of the imaginary part of the surface impedance is an intrinsic property of the PC and should not depend on the choice of field. The Zak phase of an isolated band also remains unchanged because it depends on the symmetry properties of two edge states of the band. The change of field from electric to magnetic changes the symmetry properties of both edge states and, 
therefore, keeps the Zak phase unchanged. However, the Zak phase of the 0th band will change sign, but the outcome will be the same because the effect will be canceled by the change of the factor $(-1)^{n}$ to $(-1)^{n+1}$ in Eq. (4).

\section{CONCLUSION}

In summary, we show that, due to the inversion symmetry, which is inherent in the system we are considering, the two band-edge states of any band gap have to be either symmetric or antisymmetric with respect to the chosen origin. If one band-edge state (say, the upper one) is symmetric, the other must be antisymmetric, and vice versa. The sign of the imaginary part of the impedance inside a band gap is determined by the parity of the bandedge states. As the frequency is increased from zero to the higher bands, more and more electric field oscillations appear within a unit cell. As a consequence, the parity of the eigenstate at the center or boundary of the Brillouin zone changes as we move up the bands. Our main result, i.e., Eq. (4), provides a simple and yet deterministic way to calculate the symmetry of two edge states of any particular band gap, and this is obtained by keeping track of the symmetries of all other band-edge states below the gap. We note, in particular, that the geometric Zak phase of a band tells us how the parity changes within that particular pass band. As the surface impedance determines the existence of interface states at the boundary of PCs, the existence of the interface states can be determined by the bulk band geometric phases. This correspondence between surface impedance and bulk band properties gives us a deterministic recipe to design systems with interface states.

\section{ACKNOWLEDGMENTS}

This work is supported by Hong Kong RGC through AOE/P-02/12. We thank Professor S. Q. Shen and Professor Vic Law for stimulating discussions.

\section{APPENDIX A: BANDS CROSSING CONDITION}

In the Appendixes, we give some mathematical details mentioned in the main text. We then give several additional examples in support of the statements made in the main text.

We consider a dielectric $A B$ layered structure with the relative permittivity, relative permeability, refractive index, relative impedance, and width given by $\varepsilon_{a}, \varepsilon_{b}, \mu_{a}, \mu_{b}, n_{a}$, $n_{b}, z_{a}, z_{b}, d_{a}$, and $d_{b}$ respectively, where $n_{i}=\sqrt{\varepsilon_{i}} \sqrt{\mu_{i}}$, $z_{i}=\sqrt{\mu_{i}} / \sqrt{\varepsilon_{i}}$, with $i=a, b$. The unit cell length is $\Lambda=$ $d_{a}+d_{b}$ and the relative permittivity and permeability of the slabs are positive and nondispersive. We employ several ancillary parameters $\alpha=n_{a} d_{a} /\left(n_{b} d_{b}\right), \gamma=\left(n_{a} d_{a}+\right.$ $\left.n_{b} d_{b}\right) \omega / c$, and $\tau=\left(z_{a} / z_{b}+z_{b} / z_{a}\right) / 2$. These parameters have the following physical meaning: $\alpha$ is the ratio of the optical path in slabs $A$ and $B, \gamma$ is the phase delay in a unit cell, and $\tau$ reflects the impedance mismatch between slabs
$A$ and $B$ and is always larger than 1 when the impedances of slab $A$ and slab $B$ are not the same.

Here, we prove that, when $z_{a} \neq z_{b}$, the necessary and sufficient condition for two bands to cross (either at zone center or zone boundary) is given by $\alpha=n_{a} d_{a} /\left(n_{b} d_{b}\right)$, which is a rational number, i.e., $\alpha=m_{1} / m_{2}$, where $m_{1}, m_{2} \in \mathbb{N}^{+}$.

Sufficient condition.-The band dispersion relation of the dielectric $\mathrm{AB}$ layered structure is given by [18]

$$
\begin{aligned}
\cos (q \Lambda)= & \cos k_{a} d_{a} \cos k_{b} d_{b} \\
& -\frac{1}{2}\left(\frac{z_{a}}{z_{b}}+\frac{z_{b}}{z_{a}}\right) \sin k_{a} d_{a} \sin k_{b} d_{b},
\end{aligned}
$$

where $k_{i}=\omega n_{i} / c(i=a$ or $b)$ and $q$ is the Bloch wave vector. When $\sin k_{b} d_{b}=0$, the absolute value of the rhs of Eq. (A1) is smaller than or equal to 1 , which is the frequency at which $\sin k_{b} d_{b}=0$ must be in the pass band. At $\omega=l m_{2} \pi \mathrm{c} /\left(n_{b} d_{b}\right)$, where $l \in \mathbb{N}^{+}, \quad \sin k_{b} d_{b}=0$, $\cos \left(k_{b} d_{b}\right)=(-1)^{l m_{2}}$, and $\cos \left(k_{a} d_{a}\right)=(-1)^{l m_{1}}$, so we have $\cos (q \Lambda)=(-1)^{l\left(m_{1}+m_{2}\right)}$. We get $q=0$ when $l\left(m_{1}+\right.$ $\left.m_{2}\right)$ is even, while we get $q= \pm \pi / \Lambda$ when $l\left(m_{1}+m_{2}\right)$ is odd. Near these frequencies, i.e., $\omega=\operatorname{lm}_{2} \pi \mathrm{c} /\left(n_{b} d_{b}\right)=$ $l m_{1} \pi \mathrm{c} /\left(n_{a} d_{a}\right)$, the band has linear dispersion. To prove this, we choose $l=1$ [where $\omega=m_{2} \pi \mathrm{c} /\left(n_{b} d_{b}\right)$ ] as an example; the other cases could be proved following the same process. When $m_{1}+m_{2}$ is even, the degeneracy band point is $\left(q_{0}, \omega_{0}\right)=\left[0, m_{2} \pi \mathrm{c} /\left(n_{b} d_{b}\right)\right]$. Suppose that $\left(q_{1}, \omega_{1}\right)$ is another band point near $\left(q_{0}, \omega_{0}\right)$, then $\left|q_{1}-q\right|,\left|\omega_{1}-\omega_{0}\right|$ are small numbers. Keeping to the lowest order of expansion of Eq. (A1), we have

$$
\left|q_{1}-q_{0}\right|=\sqrt{C_{1}}\left|\omega_{1}-\omega_{0}\right| / c,
$$

where

$$
C_{1}=\left[\left(n_{a} d_{a}\right)^{2}+\left(n_{b} d_{b}\right)^{2}+\left(\frac{z_{a}}{z_{b}}+\frac{z_{b}}{z_{a}}\right) n_{b} d_{b} n_{a} d_{a}\right] / \Lambda^{2} .
$$

When $m_{1}+m_{2}$ is odd, it can also be shown that $\left|q_{1}-q_{0}\right|=\sqrt{C_{1}}\left|\omega_{1}-\omega_{0}\right| / c$, where $C_{1}$ is same as before. So when $\alpha=m_{1} / m_{2}$, bands will cross at frequency points $\omega=\operatorname{lm}_{2} \pi \mathrm{c} /\left(n_{b} d_{b}\right)$ with linear dispersion.

Necessary condition.- It is easy to prove that the cross points of two bands could occur only at the boundary or the center of the BZ for 1D PC cases. If two bands cross at points other than the center or boundary of the BZ, then for frequency near the cross point, by the continuity of band dispersion, each frequency would have four corresponding Bloch vectors $q$. This is not possible because the rhs of Eq. (A1) is completely determined by the frequency and is single valued, so there can be at most two values of $q$ for each frequency. As defined previously, $\gamma \equiv\left(n_{a} d_{a}+\right.$ $\left.n_{b} d_{b}\right) \omega / c, \quad \tau \equiv \frac{1}{2}\left(z_{a} / z_{b}+z_{b} / z_{a}\right), \quad$ and $\tau>1$, when 
$z_{a} \neq z_{b}$. The rhs of Eq. (A1) can be written as $\cos \gamma-$ $(\tau-1) \sin k_{a} d_{a} \sin k_{b} d_{b}$. If $k_{a} d_{a}+k_{b} d_{b}=(2 m-1) \pi$, where $m \in \mathbb{N}^{+}$, then $\cos \gamma=-1$ and $\sin k_{a} d_{a} \sin k_{b} d_{b} \geq 0$, so the rhs of Eq. (A1) is less than or equal to -1 . If $\gamma=2 m \pi$, where $m \in \mathbb{N}^{+}$, then $\cos \gamma=1$ and $\sin k_{a} d_{a} \sin k_{b} d_{b} \leq 0$, so the rhs of Eq. (A1) is larger than or equal to 1 . In short, if one frequency satisfies the condition $\gamma=m \pi$, where $m \in \mathbb{N}^{+}$, then it must be in the band gap if two bands do not cross. If two bands cross at $\gamma=m \pi$, then $\sin k_{a} d_{a}=0$ and $\sin k_{b} d_{b}=0$ simultaneously, so $k_{a} d_{a} / k_{b} d_{b}=m_{1} \pi / m_{2} \pi=m_{1} / m_{2}$, where $m_{1}, m_{2} \in \mathbb{N}^{+}$. In other words, $\omega_{m}=m \pi c /\left(n_{a} d_{a}+n_{b} d_{b}\right)$ labels the midgap positions (or the crossing points when two bands meet) of the PC. Following the same idea, we can also prove that $\omega_{m}=(m+1 / 2) \pi c /\left(n_{a} d_{a}+n_{b} d_{b}\right)$ labels the midband positions.

\section{APPENDIX B: ZAK PHASE OF EACH BAND}

In this appendix, we show that if one isolated band (excluding the 0th band) contains the frequency point $\tilde{\omega}$ at which $\sin \left(\tilde{\omega} n_{b} d_{b} / c\right)=0$, then the Zak phase of this band must be $\pi$ (if we set the origin of the system at the center of sla $A$ ).

Proof.-The Zak phases of isolated bands depend on the choice of origin. We choose the origin to be at the center of slab $A$. To prove this assertion, we adapt the standard transfer-matrix method described in Ref. [18]. The eigenvector of the transfer matrix of the unit cell under consideration is $\left[t_{12}, \exp (i q \Lambda)-t_{11}\right]^{T}$, where $t_{11}, t_{12}$ are coefficients in the transfer matrix for one unit cell and only depend on $\omega$, and are defined as

$$
\begin{gathered}
t_{11}=\exp \left(i k_{a} d_{a}\right)\left[\cos k_{b} d_{b}+\frac{i}{2}\left(\frac{z_{a}}{z_{b}}+\frac{z_{b}}{z_{a}}\right) \sin k_{b} d_{b}\right], \\
t_{12}=\exp \left(-i k_{a} d_{a}\right)\left[\frac{i}{2}\left(\frac{z_{a}}{z_{b}}-\frac{z_{b}}{z_{a}}\right) \sin k_{b} d_{b}\right] .
\end{gathered}
$$

With this eigenvector, the eigenelectric field along the $x$ direction and the magnetic field along the $y$ direction in slab $A$ are given by

$$
\begin{aligned}
E_{x}(z)= & t_{12} \exp \left[i k_{a}\left(z+d_{a} / 2\right)\right] \\
& +\left[\exp (i q \Lambda)-t_{11}\right] \exp \left[-i k_{a}\left(z+d_{a} / 2\right)\right], \\
H_{y}(z)= & \frac{k_{a}}{\omega \mu_{a}}\left\{t_{12} \exp \left[i k_{a}\left(z+d_{a} / 2\right)\right]\right. \\
& \left.-\left[\exp (i q \Lambda)-t_{11}\right] \exp \left[-i k_{a}\left(z+d_{a} / 2\right)\right]\right\},
\end{aligned}
$$

where $t_{12}, \exp (i q \Lambda)-t_{11}$ are, respectively, the coefficients of forward wave and backward wave in slab $A$. The electric field and magnetic field in slab $B$ are given by

$$
\begin{aligned}
E_{x}(z)= & s_{11} \exp \left[i k_{b}\left(z+d_{a} / 2\right)\right] \\
& +s_{12} \exp \left[-i k_{b}\left(z+d_{a} / 2\right)\right], \\
H_{y}(z)= & \frac{k_{b}}{\omega \mu_{b}}\left\{s_{11} \exp \left[i k_{b}\left(z+d_{a} / 2\right)\right]\right. \\
& \left.-s_{12} \exp \left[-i k_{b}\left(z+d_{a} / 2\right)\right]\right\},
\end{aligned}
$$

where $s_{11}, s_{12}$ are, respectively, the coefficients of the forward wave and the backward wave in slab $B$, and the relationship between $t_{11}, t_{12}, s_{11}, s_{12}$ is given by

$$
\begin{aligned}
\left(\begin{array}{cc}
e^{i k_{b} d_{a}} & e^{-i k_{b} d_{a}} \\
e^{i k_{b} d_{a}} & -e^{-i k_{b} d_{a}}
\end{array}\right)\left(\begin{array}{c}
s_{11} \\
s_{12}
\end{array}\right)= & \left(\begin{array}{cc}
e^{i k_{a} d_{a}} & e^{-i k_{a} d_{a}} \\
\frac{z_{b}}{z_{a}} e^{i k_{a} d_{a}} & -\frac{z_{b}}{z_{a}} e^{-i k_{a} d_{a}}
\end{array}\right) \\
& \times\left(\begin{array}{c}
t_{12} \\
\exp (i q \Lambda)-t_{11}
\end{array}\right) .
\end{aligned}
$$

The mathematical details can be found in Ref. [18]. Here, we adopt some changes in notation. Knowing the eigenfield distribution, the Zak phase of each band can be further calculated with Eq. (3) in the main text. With periodic gauge, Eqs. (B2)-(B6) define the gauge for calculating the Zak phase. Below, we use this chosen gauge to prove the statement made at the beginning of this section.

We show that $t_{12}$ and $\exp (i q \Lambda)-t_{11}$ equal to zero simultaneously at the frequency point $\tilde{\omega}$ at which $\sin \left(\tilde{\omega} n_{b} d_{b} / c\right)=0$, with either $q>0$ or $q<0$. It is obvious that when $\sin \left(k_{b} d_{b}\right)=0, t_{12}=0$, and the only possible solution for $t_{12}=0$ is also $\sin \left(k_{b} d_{b}\right)=0$, when $z_{a} \neq z_{b}$ (necessary condition). Combining Eq. (A1) with the condition $\sin \left(k_{b} d_{b}\right)=0$, we have

$$
\cos [q \Lambda]=\cos \gamma
$$

where $\gamma \equiv k_{a} d_{a}+k_{b} d_{b}$ is the phase delay in each unit cell as defined before. When $\gamma \in(2 m \pi,(2 m+1) \pi)$ with $m \in \mathbb{N}, \sin [q \Lambda]=\sin \gamma$ for $q>0$. When $\gamma \in((2 m-1) \pi$, $2 m \pi)$ where $m \in \mathbb{N}, \sin [q \Lambda]=\sin \gamma$ for $q<0$. For points on the band, Eq. (A1) is automatically satisfied, so $\exp (i q \Lambda)-t_{11}$ is a pure imaginary number. And when $\sin [q \Lambda]=\sin \gamma, \operatorname{Im}\left[\exp (i q \Lambda)-t_{11}\right]=0$. So here we could conclude that $t_{12}$ and $\exp (i q \Lambda)-t_{11}$ are equal to 0 simultaneously at the frequency point where $\sin k_{b} d_{b}=0$ with either $q>0$ or $q<0$.

Suppose $t_{12}$ and $\exp (\mathrm{iq} \Lambda)-t_{11}$ are equal to 0 at $\left(q_{0}, \omega_{0}\right)$ simultaneously; thus, $\omega_{0} n_{b} d_{b} / c=m \pi, \quad m \in \mathbb{N}$. And $\left(q_{1}, \omega_{1}\right)$ is another point on the band near $\left(q_{0}, \omega_{0}\right)$, then $\omega_{1} n_{b} d_{b} / c-m \pi=\delta$, where $\delta$ is a small number. Expanding $t_{12}$ and $\exp (i q \Lambda)-t_{11}$ around $\left(q_{0}, \omega_{0}\right)$ and keep to the lowest order of $\delta$, we have 


$$
t_{12}=\exp \left(-i k_{a} d_{a}\right)\left[\frac{i}{2}\left(\frac{z_{a}}{z_{b}}-\frac{z_{b}}{z_{a}}\right)\right]\left[(-1)^{m} \delta+O^{3}(\delta)\right]
$$

$$
\exp (i q \Lambda)-t_{11} \propto O^{2}(\delta)
$$

Since the electric field is a linear combination of $t_{12}$ and $\exp (\mathrm{iq} \Lambda)-t_{11}$, it will change sign near $\left(q_{0}, \omega_{0}\right)$, i.e.,

$$
\left|u_{n q_{0}+}\right\rangle=-\left|u_{n q_{0}}\right\rangle
$$

where $\left|u_{n, q}\right\rangle$ is the normalized periodic part of the field in a cell eigenvector at $(\omega, q)$ of the $n$th band and $X^{+(-)}$ represents approaching $X$ from the positive (negative) direction.

At other band points except $\left(q_{0}, \omega_{0}\right),\left|u_{n, q}\right\rangle$ is a continuous function of $q$. Since the inversion center is chosen at the origin, the system is invariant under the space inversion. Following the same argument as stated before, we find

$$
E_{n, q}(z)=E_{n,-q}(-z)
$$

With $E_{x ; n, q}(z)=u_{n, q}(z) \exp (i q z)$, we have

$$
u_{n, q}(z)=u_{n,-q}(-z) \text {. }
$$

Since $\left|u_{n, q}\right\rangle$ is the periodic part of the wave function, the integration in $\operatorname{Im}\left\langle u_{n, q}\left|\partial_{q}\right| u_{n, q}\right\rangle$ is performed from $-\Lambda / 2$ to $\Lambda / 2$. Thus, $\operatorname{Im}\left\langle u_{n, q}\left|\partial_{q}\right| u_{n, q}\right\rangle$ is an odd function of $q$; in other words,

$$
\operatorname{Im}\left[\left\langle u_{n, q}\left|\partial_{q}\right| u_{n, q}\right\rangle+\left\langle u_{n,-q}\left|\partial_{q}\right| u_{n,-q}\right\rangle\right]=0 .
$$

If $t_{12}$ and $\exp (i q \Lambda)-t_{11}$ do not equal 0 simultaneously on one band, then with the chosen gauge, $\left|u_{n, q}\right\rangle$ is a continuous function of $q$. Thus, Eq. (B12) is applied all over that band and the Zak phase is 0 . Otherwise, if $t_{12}$ and $\exp (i q \Lambda)-t_{11}$ equal 0 simultaneously at $\left(q_{0}, \omega_{0}\right)$, then $\left|u_{n, q}\right\rangle$ is discontinuous at $\left(q_{0}, \omega_{0}\right)$, and the Zak phase of this band is given by

$$
\begin{aligned}
\gamma_{n}= & -\operatorname{Im} \lim _{\delta q \rightarrow 0}\left\{\left(\int_{-\pi / \Lambda}^{q_{0}-\delta q}+\int_{q_{0}+\delta q}^{\pi / \Lambda} d q\right)\left\langle u_{n, q}\left|\partial_{q}\right| u_{n, q}\right\rangle\right. \\
& \left.+\ln \left\langle u_{n, q_{0}+\delta q} \mid u_{n, q_{0}-\delta q}\right\rangle\right\}=\pi .
\end{aligned}
$$

The above proof can easily be extended to the case when the system is dispersive [12-14].

\section{APPENDIX C: EIGENSTATE AT THE BAND EDGE}

In this appendix, we prove that the electric field at the inversion center for a band-edge state should be zero or maximum. There are two inversion centers in this system, namely, the center of slab $A$ and slab $B$. Without loss of generality, we choose the center of slab $A$ as the inversion center. At the center of slab $A$, according to Eqs. (B3) and (B4),

$$
\begin{aligned}
& E_{x}=\zeta_{E}\left\{t_{12} \exp \left(i k_{a} d_{a}\right)+\left[\exp (i q \Lambda)-t_{11}\right]\right\}, \\
& H_{y}=\zeta_{H}\left\{t_{12} \exp \left(i k_{a} d_{a}\right)-\left[\exp (i q \Lambda)-t_{11}\right]\right\},
\end{aligned}
$$

where $\zeta_{E}$ and $\zeta_{H}$ are some complex constants. For an arbitrary state $(\omega, q)$ on the band, $t_{12} \exp \left(i k_{a} d_{a}\right)$ and $\exp (i q \Lambda)-t_{11}$ are pure imaginary numbers. At band edges, $\cos (q \Lambda)= \pm 1, \sin (q \Lambda)=0$. After some mathematics, we could arrive at

$$
\begin{aligned}
& \{\text { rhs of Eq. }(\mathrm{A} 1)\}^{2}+\left\{\operatorname{Im}\left[\exp (i q \Lambda)-t_{11}\right]\right\}^{2} \\
& \quad=1+\left\{\operatorname{Im}\left[t_{12} \exp \left(i k_{a} d_{a}\right)\right]\right\}^{2}
\end{aligned}
$$

then

$$
t_{12} \exp \left(i k_{a} d_{a}\right)= \pm\left[\exp (i q \Lambda)-t_{11}\right] .
$$

From Eq. (C4), it is easy to find that either the electric field or the magnetic field should be 0 at the center of slab $A$. So there are only two types of states at the band edges. For the type-A (antisymmetry) state, $E_{x}(z=0)=0$. For the type$S$ (symmetry) state, $E_{x}(z=0) \neq 0, H_{y}(z=0)=0$; the electric field is at the maximum value inside slab $A$.

Now we go further to find out whether the $A$ or the $S$ state is at the lower or upper edge of the $n$th gap. The sign of the function $\sin \left(\omega n_{b} d_{b} / c\right)$ depends on the number of zeros it crosses in the frequency range $(0, \omega)$, so to get $\operatorname{sgn}\left(\sin k_{b} d_{b}\right)$, we only need to count the number of zero points of $\sin k_{b} d_{b}$. As proved before, if the frequency $\tilde{\omega}$ at which $\sin k_{b} d_{b}=0$ is on an isolated band, then this band has Zak phase $\pi$; otherwise, $\tilde{\omega}$ is at the crossing point of two bands since $\tilde{\omega}$ is always on the pass band. Thus, for a frequency $\omega$ inside the $n$th gap,

$$
\operatorname{sgn}\left(\sin \frac{\omega}{c} n_{b} d_{b}\right)=(-1)^{l} \exp \left(i \sum_{m=1}^{n-1} \theta_{m}^{\mathrm{Zak}}\right)
$$

where $l \in \mathbb{N}$ is the number of band crossing points under the $n$th gap, and the second term on the rhs of Eq. (C5) is a summation of the Zak phase below this gap. We then define

$$
\chi=\operatorname{sgn}\left(1-\varepsilon_{a} \mu_{b} / \varepsilon_{b} \mu_{a}\right)
$$

according to Eq. (B2): 


$$
\operatorname{sgn}\left\{\operatorname{Im}\left[t_{12} \exp \left(i k_{a} d_{a}\right)\right]\right\}=(-1)^{l} \exp \left(i \sum_{m=1}^{n-1} \theta_{m}^{\mathrm{Zak}}\right) \chi
$$

With Eq. (B1), it is easy to get, for the band-edge states,

$$
\operatorname{Im}\left[\exp (i q \Lambda)-t_{11}\right]=-\left[\sin (\gamma)+(\tau-1) \cos k_{a} d_{a} \sin k_{b} d_{b}\right] .
$$

At $\quad \gamma=(2 n+1 / 2) \pi, \quad$ where $\quad n \in \mathbb{N}, \quad \sin (\gamma)=1$, $\cos k_{a} d_{a} \sin k_{b} d_{b}=\cos ^{2}\left(k_{a} d_{a}\right) \geq 0$; at $\gamma=(2 n+3 / 2) \pi$, where $\quad n \in \mathbb{N}, \quad \sin (\gamma)=-1, \quad \cos k_{a} d_{a} \sin k_{b} d_{b}=$ $-\cos ^{2}\left(k_{a} d_{a}\right) \leq 0$. Thus, at $\gamma=(n+1 / 2) \pi$, where $n \in \mathbb{N}$,

$$
\operatorname{sgn}\left[\sin (\gamma)+(\tau-1) \cos k_{a} d_{a} \sin k_{b} d_{b}\right]=\operatorname{sgn}[\sin (\gamma)] .
$$

From Eq. (C3), it is easy to get that, for states on the band,

$$
\begin{aligned}
& {\left[\sin (\gamma)+(\tau-1) \cos k_{a} d_{a} \sin k_{b} d_{b}\right]^{2}} \\
& \quad=\left(\tau^{2}-1\right) \sin ^{2} k_{b} d_{b}+1-\cos (q \Lambda) \geq 0 .
\end{aligned}
$$

The equality is achieved at the point where two bands cross; thus, $\left[\sin (\gamma)+(\tau-1) \cos k_{a} d_{a} \sin k_{b} d_{b}\right]$ does not change sign on the isolated pass band. $\sin (\gamma)$ changes sign only inside the band gap (or at the crossing point of two bands) and the frequency at which $\gamma=(n+1 / 2) \pi$ must be in the pass band, so Eq. (C9) is also true for band-edge states. As $\gamma=n \pi\left(n \in \mathbb{N}^{+}\right)$gives the midgap position of the $n$th gap, for the edge state below the $n$th gap,

$$
\operatorname{sgn}\left\{\operatorname{Im}\left[\exp (i q \Lambda)-t_{11}\right]\right\}=-\operatorname{sgn}[\sin (\gamma)]=(-1)^{n},
$$

for the edge state above the $n$th gap,

$$
\operatorname{sgn}\left\{\operatorname{Im}\left[\exp (i q \Lambda)-t_{11}\right]\right\}=(-1)^{n+1} .
$$

Therefore, if $(-1)^{n}(-1)^{l} \exp \left(i \sum_{m=1}^{n-1} \theta_{m}^{\mathrm{Zak}}\right) \chi=1$, then the edge state below the $n$th gap is an $S$ state, and above the $n$th gap is the $A$ state; otherwise, if $(-1)^{n}(-1)^{l} \exp \left(i \sum_{m=1}^{n-1} \theta_{m}^{\mathrm{Zak}}\right) \chi=-1$, then the state below the $n$th gap is an $A$ state, and above the $n$th gap is an $S$ state.

In Fig. 5, we give an example to illustrate the relation between the edge state and the Zak phase. The band structure (solid black line) of a particular PC with parameters given by $\varepsilon_{a}=4, \mu_{a}=\varepsilon_{b}=\mu_{b}=1, d_{a}=0.4 \Lambda$, and $d_{b}=0.6 \Lambda$ is plotted in Fig. 5. The rule specified in Appendix B gives the Zak phase of each isolated band, as shown in green. We labeled the type-A edge states with solid purple circles and the $S$ state with yellow circles. As in Fig. 1(b), $\operatorname{sgn}[\varsigma]=\operatorname{sgn}\left[\operatorname{Im}\left(Z_{s}\right)\right]$ is marked by magenta when $\varsigma>0$ and cyan when $\varsigma<0$. We point out three important features of Fig. 5: (i) The state must change from

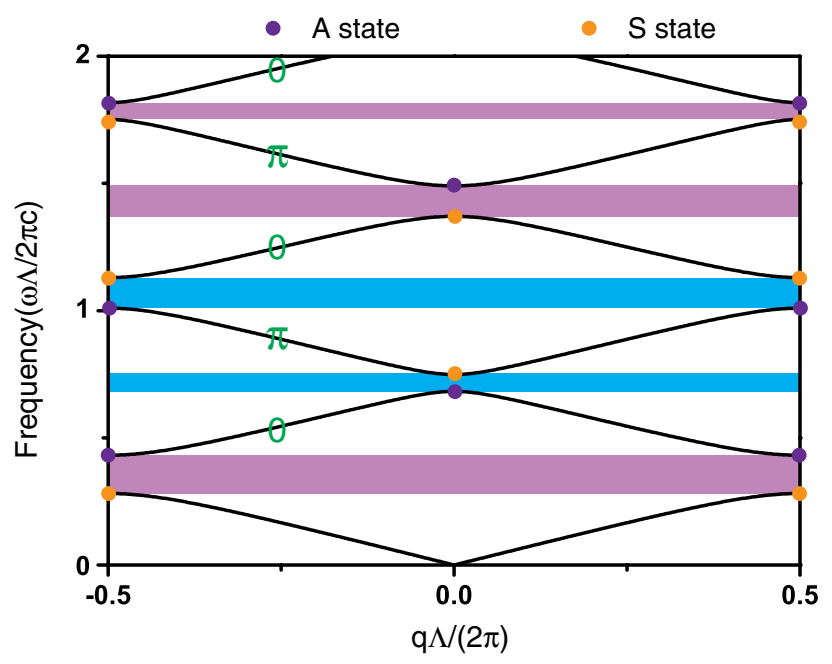

FIG. 5. The band structure (solid black line) of the PC with parameters given by $\varepsilon_{a}=4, \mu_{a}=\varepsilon_{b}=\mu_{b}=1, d_{a}=0.4 \Lambda$, and $d_{b}=0.6 \Lambda$.The light magenta strip represents the gap with $\varsigma>0$, while the cyan strip represents the gap with $\varsigma<0$, the Zak phase of each individual band is labeled in green. The solid purple circle is the $A$ (antisymmetric) state $\left(E_{\text {eigen }}=0\right.$ at the center of slab $A$ ) at the band edge, and the solid yellow circle is the $S$ (symmetric) state $\left(E_{\text {eigen }} \neq 0\right.$ at the center of slab $\left.A\right)$.

$(S)$ to $(A)$ or from $(A)$ to $(S)$ when passing through a band gap, (ii) the upper and lower edge states of a band are of the same type if the Zak phase of this band is 0, otherwise it is $\pi$, and (iii) $\operatorname{sgn}[\varsigma]$ of each gap is related to the edge state bounding this gap. If the $(A)$ state is at the lower edge and the $(S)$ state is at the upper edge, then $\varsigma<0$; otherwise, if the $(S)$ state is at the lower edge and the $(A)$ states is at the upper edge, $\varsigma>0$. This will be proved in Appendix D.

\section{APPENDIX D: BULK BAND AND SIGN OF REFLECTION PHASE}

In this appendix, we show that

$$
\operatorname{sgn}\left(\phi_{n}\right)=(-1)^{n}(-1)^{l} \exp \left(i \sum_{m=1}^{n-1} \theta_{m}^{\mathrm{Zak}}\right) \chi,
$$

where $\phi_{n}$ is the reflection phase of the $n$th gap (as defined below).

We consider a plane wave $E_{i}=E_{0} e^{i k z}$ being incident on the $\mathrm{PC}$ from vacuum as shown in Fig. 1(a), and the reflected wave is $E_{r}=r E_{0} e^{-i k z}$. The field inside the gap at $z=0^{+}$is given by

$$
\begin{aligned}
E_{x}= & t_{12} \exp \left(i k_{a} d_{a} / 2\right)+\left[\exp (i q \Lambda)-t_{11}\right] \exp \left(-i k_{a} d_{a} / 2\right), \\
H_{y}= & \frac{k_{a}}{\omega \mu_{a}}\left\{t_{12} \exp \left(i k_{a} d_{a} / 2\right)\right. \\
& \left.-\left[\exp (i q \Lambda)-t_{11}\right] \exp \left(-i k_{a} d_{a} / 2\right)\right\} .
\end{aligned}
$$


Matching the boundary condition, we have

$$
\frac{1+r}{1-r}=z_{a} \frac{t_{12} \exp \left(i k_{a} d_{a}\right)+\left[\exp (i q \Lambda)-t_{11}\right]}{t_{12} \exp \left(i k_{a} d_{a}\right)-\left[\exp (i q \Lambda)-t_{11}\right]}
$$

From Eq. (D4), we calculate the reflection phase delay inside the gap. As an example, in Fig. 6, we give the reflection phase delay inside the first gap of $\mathrm{PC} 3\left(\varepsilon_{b}=3.5\right.$, $\varepsilon_{a}=\mu_{a}=\mu_{b}=1, d_{a}=0.35 \Lambda$, and $d_{b}=0.65 \Lambda$ ) for light incident from vacuum (black line). The reflection phase increases monotonically from $-\pi$ to 0 with increasing frequency. As a comparison, we also give the reflection phase calculated directly using the transfer matrix for a slab consisting of a finite number of unit cells of PC3. The solid blue line is for a slab with 5 unit cells and the solid red line is for a slab with 10 unit cells of PC3. As the number of unit cells increases, the reflection phase converges to the one given by Eq. (D4), which is derived for a semi-infinite PC.

For frequency inside the $n$th gap (including band-edge states),

$$
q \Lambda=n \pi+i \mathrm{~K}^{18}
$$

where $\mathrm{K}>0$ and describes the decay length inside the gap.

$$
\begin{aligned}
& \left|t_{12} \exp \left(i k_{a} d_{a}\right)\right|^{2}-\left|\operatorname{Im}\left[\exp (i q \Lambda)-t_{11}\right]\right|^{2} \\
& \quad=\cosh ^{2}(\mathrm{~K})-1 \geq 0,
\end{aligned}
$$

and the equality is achieved only at the band edge. From Eq. (B2), we know $t_{12} \exp \left(i k_{a} d_{a}\right)$ is a pure imaginary number, so

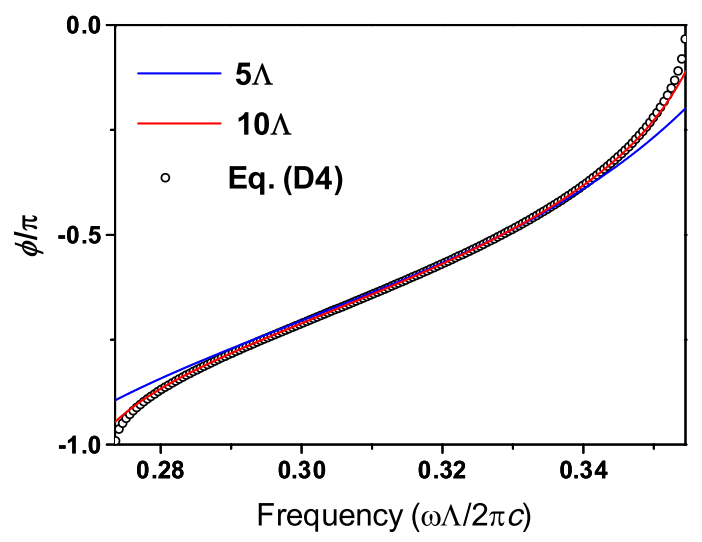

FIG. 6. The reflection phase of PC3 in vacuum inside the first band gap. The parameters of PC3 are given by $\varepsilon_{b}=3.5$, $\varepsilon_{a}=\mu_{a}=\mu_{b}=1, d_{a}=0.35 \Lambda$, and $d_{b}=0.65 \Lambda$. The solid blue line and the solid red line are calculated with the transfer matrix directly and for 5 and 10 unit cells of PC3, respectively. The open black circle is calculated with the Eq. (D4). It is clear that, as the unit number increase, the reflection phase converges to the one calculated with the Eq. (D4).

$$
\begin{aligned}
& \operatorname{sgnIm}\left\{t_{12} \exp \left(i k_{a} d_{a}\right) \pm\left[\exp (i q \Lambda)-t_{11}\right]\right\} \\
& \quad=\operatorname{sgnIm}\left[t_{12} \exp \left(i k_{a} d_{a}\right)\right]=(-1)^{l} \exp \left(i \sum_{m=1}^{n-1} \theta_{m}^{\mathrm{Zak}}\right) \chi .
\end{aligned}
$$

Inside the $n$th gap,

$$
\operatorname{Re}\left[\exp (i q \Lambda)-t_{11}\right]=(-1)^{n+1} \sinh K .
$$

So,

$$
\frac{1+r}{1-r}=z_{a} \frac{(-1)^{n}(-1)^{l} \exp \left(i \sum_{m=1}^{n-1} \theta_{m}^{\mathrm{Zak}}\right) \chi \Lambda_{+}+i}{(-1)^{n}(-1)^{l} \exp \left(i \sum_{m=1}^{n-1} \theta_{m}^{\mathrm{Zak}}\right) \chi \Lambda_{-}-i}
$$

where

$\lambda_{ \pm}=\left|t_{12} \exp \left(i k_{a} d_{a}\right) \pm i \operatorname{Im}\left[\exp (i q \Lambda)-t_{11}\right]\right| / \sinh K>0$.

Inside the $n$th gap, $r=e^{i \phi_{n}}$, where $\phi_{n}$ (the subscript $\mathrm{n}$ labels the gap) is a function of frequency. Thus $(1+r) /(1-r)=i \cot \left(\phi_{n} / 2\right)$, a pure imaginary number, which means the rhs of Eq. (D9) is also pure imaginary. After some mathematical works, we can prove $\lambda_{+} \lambda_{-}=1$ and

$$
\begin{aligned}
& \operatorname{Im}\left[z_{a} \frac{(-1)^{n}(-1)^{l} \exp \left(i \sum_{m=1}^{n-1} \theta_{m}^{\mathrm{Zak}}\right) \chi \lambda_{+}+i}{(-1)^{n}(-1)^{l} \exp \left(i \sum_{m=1}^{n-1} \theta_{m}^{\mathrm{Zak}}\right) \chi \lambda_{-}-i}\right] \\
& \quad=\operatorname{sgn}\left[(-1)^{n}(-1)^{l} \exp \left(i \sum_{m=1}^{n-1} \theta_{m}^{\mathrm{Zak}}\right) \chi\right] z_{a} \lambda_{+} .
\end{aligned}
$$

So if the reflection phase is limited to $[-\pi, \pi]$ and from the relation $\phi_{n}=\pi-2 \arctan \left(\varsigma_{n}\right)$ (here we shift $\phi_{n}$ back to be inside $[-\pi, \pi])$, then

$$
\operatorname{sgn}\left[\varsigma_{n}\right]=\operatorname{sgn}\left(\phi_{n}\right)=(-1)^{n}(-1)^{l} \exp \left(i \sum_{m=1}^{n-1} \theta_{m}^{\mathrm{Zak}}\right) \chi .
$$

It is easy to show that $\operatorname{Im}\left[\exp (i q \Lambda)-t_{11}\right]$ is a monotonic function inside the $n$th gap. As $\lambda_{+} \lambda_{-}=1$, and the difference between $\lambda_{+}, \lambda_{-}$is $\operatorname{Im}\left[\exp (i q \Lambda)-t_{11}\right]$; thus, $\lambda_{+}$is a monotonic function inside the $n$th gap. Combining Eqs. (D9) and (D11), we that find $\phi_{n}$ is also a monotonic function. As we already proved, the edge state must be an $A$ state or an $S$ state. When A is present, $r=-1$ and $\phi= \pm \pi$; when $S$ is present, $r=1$ and $\phi=0$. With Eq. (D11) and the reflection phase at the band edge, we further conclude that $\phi_{n}$ is a monotonic increasing function of frequency. From the relation $\phi_{n}=\pi-2 \arctan \left(\varsigma_{n}\right)$, it is straight forward to show that $\varsigma_{n}$ is a monotonic decreasing function of 


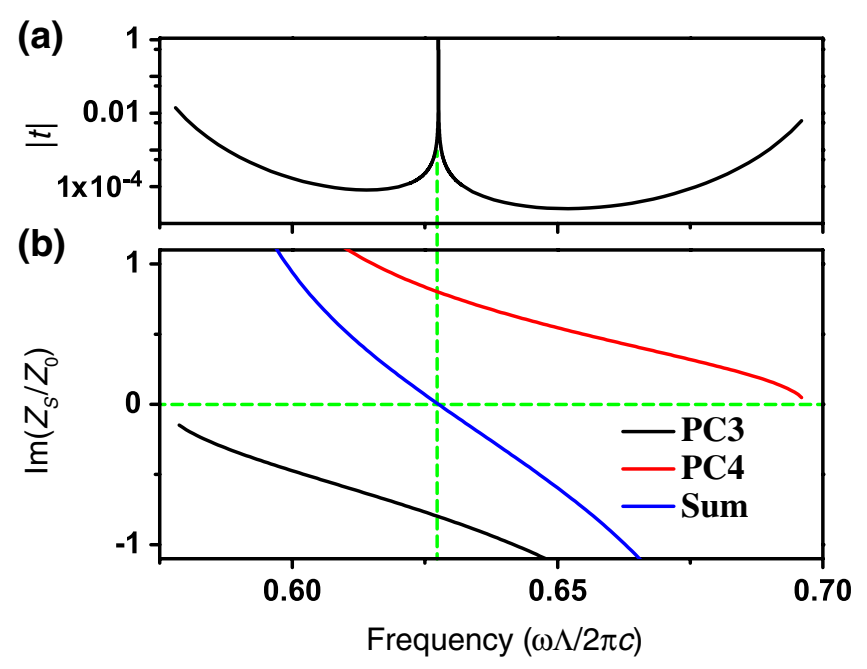

FIG. 7. (a) The transmission spectrum of the system composed of 10 unit cells of PC3 on the left-hand side and 10 unit cells of PC4 on the right-hand side in vacuum. The parameters of PC3 are given by $\varepsilon_{b}=3.5, \quad \varepsilon_{a}=\mu_{a}=\mu_{b}=1, \quad d_{a}=0.35 \Lambda$, and $d_{b}=0.65 \Lambda$, and the parameters of PC4 are given by $\mu_{b}=6$, $\varepsilon_{a}=\mu_{a}=\varepsilon_{b}=1, d_{a}=0.6 \Lambda$, and $d_{b}=0.4 \Lambda$, where $\Lambda$ is the unit length of PCs. (b) The imaginary part of relative surface impedance (divided by the impedance of vacuum) of PC3 (solid black line), PC4 (solid red line), and the sum of the two (solid blue line) inside the common gap region. The green dashed lines are for illustration only; the position where the blue line crosses 0 corresponds to an interface state between two PCs.

frequency from $\infty$ to 0 or from 0 to $-\infty$ depending on the sign of $\varsigma_{n}$.

In the main text, we argue that, once sgns of the left and right PCs are different inside the common band gap, there must be an interface state; here, we give another example. In Fig. 7(b), we choose the 2nd common band gap in Fig. 4 as an example. The solid black, red, and blue lines show the imaginary parts of relative impedances of PC3, PC4, and the sum of those two, respectively, inside the 2nd common band gap. The solid black and red lines are both monotonic decreasing functions of frequency, and their sum must also be a monotonic decreasing function of frequency from positive to negative. Thus, there must exist some frequency point at which the blue line crosses 0 , corresponding to an interface state, as shown in Fig. 7(a), where resonant transmission is observed inside the common band gap.

\section{APPENDIX E: EXTENSION OF EQ. (4)}

In this appendix, we show that Eq. (4) is still valid when the relative permittivity and permeability are continuously varying functions, and the lattice constants of the left and right periodic systems do not need to be equal.

For an interface state to exist, we only need two overlapped gaps with different signs of $\varsigma=\operatorname{Im}\left[Z_{S} / Z_{0}\right]$ and we do not care about the "origin" of the gap (e.g., gap number

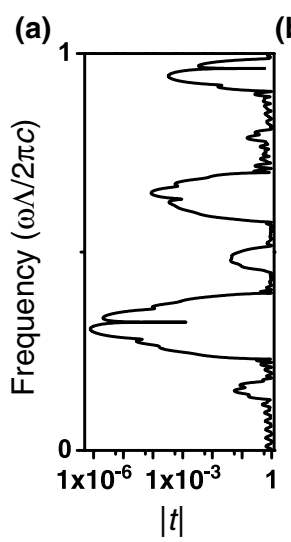

(b)

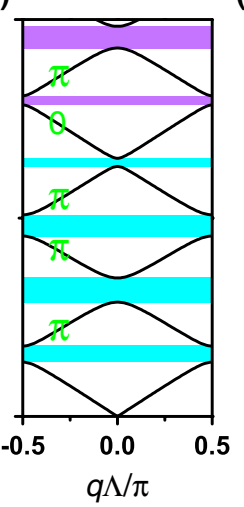

(c)

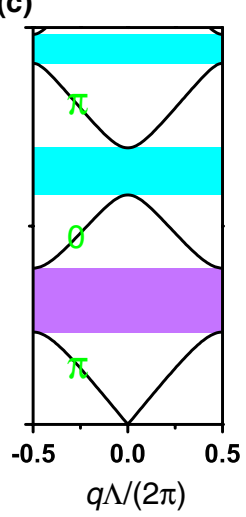

FIG. 8. (a) The transmission spectrum of the system constructed with 7 unit cells of PC5 on the left-hand side and 14 unit cells of PC4 on the right-hand side in vacuum. The parameters of PC5 are given by $\varepsilon_{b}=3.5, \varepsilon_{a}=\mu_{a}=\mu_{b}=1, d_{a}=0.7 \Lambda$, and $d_{b}=1.3 \Lambda$, which just doubles the length of PC 3 ; the parameters of PC4 are given by $\mu_{b}=6, \varepsilon_{a}=\mu_{a}=\varepsilon_{b}=1, d_{a}=0.6 \Lambda$, and $d_{b}=0.4 \Lambda$. (b),(c) The band structure (solid black curve) of PC5 and PC4. In both (b) and (c), the magenta strip represents the gap with $\varsigma>0$, while the cyan strip represents the gap with $\varsigma<0$, and the Zak phase of each individual band is also labeled in (b) and (c) in green.

or the lattice constants of PCs of the left or right periodic system). In Fig. 8, we give an example to illustrate this point. We consider a system consisting of 7 unit cells of PC5 $\quad\left(\varepsilon_{b}=3.5, \quad \varepsilon_{a}=\mu_{a}=\mu_{b}=1, \quad d_{a}=0.7 \Lambda, \quad\right.$ and $d_{b}=1.3 \Lambda$ ) on the left and 14 unit cells of PC4 on the right embedded in vacuum. The parameters of PC4 are the

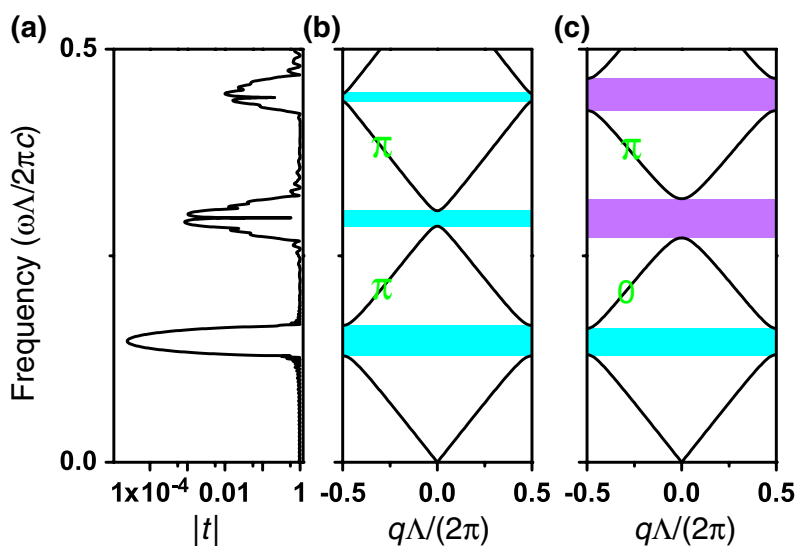

FIG. 9. (a) The transmission spectrum of the system constructed with 20 unit cells of PC6 on the left-hand side and 10 unit cells of PC7 on the right-hand side in vacuum, where the parameters of PC6 are given by $\varepsilon_{r}=12+6 \sin [2 \pi(\mathrm{z} / \Lambda+$ 1/4)], $\mu_{r}=1$, and the parameters of PC7 are given by $\varepsilon_{r}=12+5 \sin [2 \pi(\mathrm{z} / \Lambda-1 / 4)]+5 \sin [4 \pi(\mathrm{z} / \Lambda+1 / 8)], \quad \mu_{r}=1$. The boundary between PC6 and PC7 is now set at $z=0$. (b), (c) The band structure (solid black line) of PC6 and PC7. In both (b) and (c), the magenta strip represents the gap with $\varsigma>0$, while the cyan strip represents the gap with $\varsigma<0$, and the Zak phase of each individual band is also labeled in (b) and (c) in green. 
same as that in the main text; i.e., $\mu_{b}=6, \varepsilon_{a}=\mu_{a}=$ $\varepsilon_{b}=1, d_{a}=0.6 \Lambda$, and $d_{b}=0.4 \Lambda$. Note here that we just double the length of $d_{a}$ and $d_{b}$ of PC3, so that the lattice constant of PC5 is twice that of PC4. According to the scaling law, the Zak phase of each isolated band would not change, as labeled in Figs. 8(b) and 8(c) in green. Using Eq. (4), we get $\operatorname{sgn}[\varsigma]$, which is also labeled with magenta $(\varsigma>0)$ or cyan $(\varsigma<0)$ in Figs. 8(b) and 8(c). Though now the gap numbers of PCs from the left-hand and righthand sides of the common gap region are different, the rule still applies, because whenever two gaps with different color have common frequency region, there must be an interface state.

Equation (4) also applies when the dielectric function is a continuous function of $z$. In Fig. 9, we consider a system that consists of 20 unit cells of PC6 $\left(\varepsilon_{r}=\right.$ $\left.12+6 \sin [2 \pi(\mathrm{z} / \Lambda+1 / 4)], \mu_{r}=1\right)$ on the left-hand side and 10 unit cells of PC7 $\left[\varepsilon_{r}=12+5 \sin [2 \pi(\mathrm{z} / \Lambda-1 / 4)]+\right.$ $\left.5 \sin [4 \pi(\mathrm{z} / \Lambda+1 / 8)], \quad \mu_{r}=1\right]$ on the right-hand side embedded in vacuum. The transmission spectrum of the system is given in Fig. 9(a), where the boundary between two PCs is set at $z=0$. The band structures (solid black line) of PC6 and PC7 are given in Figs. 9(b) and 9(c), respectively. We calculate the Zak phase of each band numerically with Eq. (3) and label them in green, then $\operatorname{sgn}[\varsigma]$ of each gap is also shown in magenta $(\varsigma>0)$ or cyan $(\varsigma<0)$ in Figs. 9(b) and 9(c). It is clear that Eq. (4) could still predict the existence or absence of the interface state in this case.

[1] J. Zak, Berry's Phase for Energy Bands in Solids, Phys. Rev. Lett. 62, 2747 (1989).

[2] Y. Hatsugai, Chern Number and Edge States in the Integer Quantum Hall Effect, Phys. Rev. Lett. 71, 3697 (1993).

[3] Y. Hatsugai, T. Fukui, and H. Aoki, Topological Analysis of the Quantum Hall Effect in Graphene: Dirac-Fermi Transition across van Hove Singularities and Edge versus Bulk Quantum Numbers, Phys. Rev. B 74, 205414 (2006).

[4] M. S. Rudner, N. H. Lindner, E. Berg, and M. Levin, Anomalous Edge States and the Bulk-Edge Correspondence for Periodically Driven Two-Dimensional Systems, Phys. Rev. X 3, 031005 (2013).

[5] N. Regnault and B.A. Bernevig, Fractional Chern Insulator, Phys. Rev. X 1, 021014 (2011).
[6] W. P. Su, J. R. Schrieffer, and A. J. Heeger, Solitons in Polyacetylene, Phys. Rev. Lett. 42, 1698 (1979).

[7] W. P. Su, J. R. Schrieffer, and A. J. Heeger, Soliton Excitations in Polyacetylene, Phys. Rev. B 22, 2099 (1980).

[8] A. J. Heeger, S. Kivelson, J. R. Schrieffer, and W. P. Su, Solitons in Conducting Polymers, Rev. Mod. Phys. 60, 781 (1988).

[9] M.Z. Hasan and C. L. Kane, Colloquium: Topological Insulators, Rev. Mod. Phys. 82, 3045 (2010).

[10] B. A. Bernevig, T. L. Hughes, and S.-C. Zhang, Quantum Spin Hall Effect and Topological Phase Transition in HgTe Quantum Wells, Science 314, 1757 (2006).

[11] O. A. Pankratov, S. V. Pakhomov, and B. A. Volkov, Supersymmetry in Heterojunctions: Band-Inverting Contact on the Basis of Pb1_xSnxTe and Hg1_xCdxTe, Solid State Commun. 61, 93 (1987).

[12] F. D. M. Haldane and S. Raghu, Possible Realization of Directional Optical Waveguides in Photonic Crystals with Broken Time-Reversal Symmetry, Phys. Rev. Lett. 100, 013904 (2008).

[13] S. Raghu and F. D. M. Haldane, Analogs of Quantum-HallEffect Edge States in Photonic Crystals, Phys. Rev. A 78, 033834 (2008).

[14] A. Raman and S. Fan, Photonic Band Structure of Dispersive Metamaterials Formulated as a Hermitian Eigenvalue Problem, Phys. Rev. Lett. 104, 087401 (2010).

[15] W. Tan, Y. Sun, H. Chen, and S.-Q. Shen, Photonic Simulation of Topological Excitations in Metamaterials, arXiv:1211.5413.

[16] F. J. Lawrence, L. C. Botten, K. B. Dossou, C. M. de Sterke, and R. C. McPhedran, Impedance of Square and Triangular Lattice Photonic Crystals, Phys. Rev. A 80, 023826 (2009).

[17] F. J. Lawrence, L. C. Botten, K. B. Dossou, R. C. McPhedran, and C. M. de Sterke, Photonic-Crystal Surface Modes Found from Impedances, Phys. Rev. A 82, 053840 (2010).

[18] A. Yariv and P. Yeh, Optical Waves in Crystals: Propagation and Control of Laser Radiation (Wiley, New York, 1984).

[19] R. Raffaele, Manifestations of Berry's Phase in Molecules and Condensed Matter, J. Phys. Condens. Matter 12, R107 (2000).

[20] W. Kohn, Analytic Properties of Bloch Waves and Wannier Functions, Phys. Rev. 115, 809 (1959).

[21] R. L. Liboff, Introductory Quantum Mechanics (AddisonWesley, Reading, MA, 1992), 4th ed. 